\title{
Oxygen vacancies in strontium titanate: A DFT+DMFT study
}

\author{
Jaime Souto-Casares, ${ }^{*}$ Nicola A. Spaldin $\odot,{ }^{\dagger}$ and Claude Ederer $\odot^{\ddagger}$ \\ Materials Theory, ETH Zürich, Wolfgang-Pauli-Strasse 27, 8093 Zürich, Switzerland
}

(Received 17 December 2020; accepted 16 March 2021; published 9 April 2021)

\begin{abstract}
We address the long-standing question of the nature of oxygen vacancies in strontium titanate, using a combination of density functional theory and dynamical mean-field theory (DFT+DMFT) to investigate in particular the effect of vacancy-site correlations on the electronic properties. Our approach uses a minimal low-energy electronic subspace including the Ti- $t_{2 g}$ orbitals plus an additional vacancy-centered Wannier function, and it provides an intuitive and physically transparent framework to study the effect of the local electron-electron interactions on the excess charge introduced by the oxygen vacancies. We estimate the strength of the screened interaction parameters using the constrained random phase approximation, and we find a sizable Hubbard $U$ parameter for the vacancy orbital. Our main finding, which reconciles previous experimental and computational results, is that the ground state is either a state with double occupation of the localized defect state or a state with a singly occupied vacancy and one electron transferred to the conduction band. The balance between these two competing states is determined by the strength of the interaction both on the vacancy and the Ti sites, and on the Ti-Ti distance across the vacancy. Finally, we contrast the case of vacancy doping in $\mathrm{SrTiO}_{3}$ with doping via $\mathrm{La}$ substitution, and we show that the latter is well described by a simple rigid-band picture.
\end{abstract}

DOI: 10.1103/PhysRevResearch.3.023027

\section{INTRODUCTION}

Strontium titanate, $\mathrm{SrTiO}_{3}$, is a perovskite-structure oxide with the ideal cubic $\operatorname{Pm} \overline{3} m$ structure at room temperature, and a band-insulating electronic structure due to the formal $3 d^{0}$ configuration of the $\mathrm{Ti}^{4+}$ cations. In spite of its apparent simplicity, $\mathrm{SrTiO}_{3}$ shows a wealth of interesting and sometimes technologically relevant properties, such as tunability of its high dielectric constant [1,2], quantum paraelectricity $[3,4]$, and even superconductivity [5]. Although these properties have been known for many years, there remain many open questions [6]. For example, superconductivity occurs at unusually low doping levels [7], indicating an exotic mechanism possibly related to its quantum paraelectricity [8]. At the same time, reports of a two-dimensional electron gas [9] and emergent magnetism [10] at surfaces and interfaces have rekindled interest in $\mathrm{SrTiO}_{3}$ thin films for oxide electronics.

All of these phenomena require the existence of electronic charge carriers, which are usually introduced through oxygen vacancies $\left(\mathrm{O}_{\mathrm{V}}\right)$ [11], substitution of $\mathrm{Sr}^{2+}$ by a trivalent ion such as $\mathrm{La}^{3+}$, or of $\mathrm{Ti}^{4+}$ by a pentavalent ion such as $\mathrm{Nb}^{5+}$. Interestingly, the resulting properties can be quite sensitive to the specific type of doping [12-22]. Regarding oxygenvacancy doping in $\mathrm{SrTiO}_{3}$, perhaps the most pressing open

\footnotetext{
*jaime.soutocasares@mat.ethz.ch

†nicola.spaldin@mat.ethz.ch

‡claude.ederer@mat.ethz.ch
}

Published by the American Physical Society under the terms of the Creative Commons Attribution 4.0 International license. Further distribution of this work must maintain attribution to the author(s) and the published article's title, journal citation, and DOI. question is the nature of the introduced charge, with different experimental measurements leading to apparently contradictory conclusions. On the one hand, it is known that even very low concentrations of oxygen vacancies cause metallicity, with transport measurements indicating an increase in carrier density with increasing oxygen vacancy concentration [23-25]. On the other hand, there are multiple reports of optical absorption signals within the band gap [26-29], such as, for example, a red luminescence at $2.0 \mathrm{eV}$, attributed to localized electrons forming $\mathrm{Ti}^{3+}$ polarons trapped at isolated oxygen vacancies [30].

Computational studies aiming to clarify the physics of the $\mathrm{O}_{\mathrm{V}}$ state also show a range of conflicting scenarios, largely due to their different treatments of exchange and correlation effects. (For a detailed summary, see Ref. [31].) Standard density functional calculations using the local density approximation (LDA) or generalized gradient approximation (GGA) predict a delocalized defect state at the bottom of the Ti- $t_{2 g}$ conduction band, consistent with the observed metallicity [32-34]. Use of B3PW hybrid functionals or the LDA $+\mathcal{U}$ method, however, lead to a doubly occupied in-gap state, $0.77 \mathrm{eV}(\mathrm{B} 3 \mathrm{PW})$ or $0.11 \mathrm{eV}(\mathrm{LDA}+\mathcal{U}$, with $\mathcal{U}=5 \mathrm{eV})$ below the minimum of the conduction band, but do not capture the reported metallic conduction (although this can be reconciled by considering the formation of polarons) [35]. Using spin-polarized GGA $+\mathcal{U}$ calculations with $\mathcal{U}=5.0 \mathrm{eV}$, the two electrons released by the missing oxygen are found to distribute between a localized magnetic in-gap state and a delocalized state in the conduction band, consistent with both sets of reported experimental behaviors [31]. Finally, we mention a recent DFT $+\mathcal{U}+\mathcal{V}$ study, including selfconsistent on-site and intersite electronic interactions, which gives a good description of both stoichiometric and oxygendeficient STO, with the details of the band structure and the 
vacancy formation energies agreeing well with experiments [36]. The authors also present a systematic study of the effects of various parameters such as structure and cell size, the used exchange-correlation functional, and the treatment of structural relaxations and spin polarization on the resulting properties.

These previous studies clearly indicate that electron interaction effects are important in describing the behavior of oxygen vacancies in $\mathrm{SrTiO}_{3}$ [37], in spite of the bandinsulating nature of the stoichiometric host material. However, while such DFT $+\mathcal{U}(+\mathcal{V})$ calculations are able to appropriately treat strong interactions between electrons in the transition-metal $d$ states (and in principle the oxygen $p$ states), this is usually achieved by introducing an artificial symmetry breaking resulting in long-range magnetic order. Furthermore, current implementations of the $\mathrm{DFT}+\mathcal{U}$ formalism are typically based on projections on site-centered atomic orbitals, and so they leave electrons at the vacancy site uncorrelated. The importance of explicitly considering interactions on the vacancy site was shown in model calculations using a minimal three-orbital model representing the vacancy, coupled to a bath representing the $t_{2 g}$ bulk bands [38], but a full first-principles description is still lacking.

Here, we study the electronic properties of oxygendeficient $\mathrm{SrTiO}_{3}$ using a combination of density functional theory (DFT) and dynamical mean-field theory (DMFT). The latter accounts for all dynamic correlation effects between electrons on the same "site" and thus introduces genuine many-body effects in the electronic structure obtained from DFT, and it has already been applied recently to study oxygen vacancy complexes at the $\mathrm{SrTiO}_{3}$ surface [39]. Similar to our previous work on oxygen-deficient $\mathrm{LaTiO}_{3}$ [40], we focus on the most important bands at the bottom of the conduction band, which can be expressed in a basis of maximally localized Wannier functions (MLWFs) $[41,42]$ with predominant Ti- $t_{2 g}$ character plus one additional Wannier function located at the vacancy site. This results in a physically intuitive framework to study the distribution of charge between the vacancy site and the Ti- $t_{2 g}$ conduction bands.

We estimate the strength of the screened electron-electron interaction, both for the Ti- $t_{2 g}$ orbitals and for the vacancy level, using the constrained random phase approximation (cRPA) [43-46]. We obtain a sizable $U$ parameter for the vacancy level, confirming that the corresponding correlation effects should not be ignored. We then find that the occupation of the vacancy site is indeed controlled by the strength of the electron-electron repulsion both on the $\mathrm{O}_{\mathrm{V}}$ as well as on the Ti sites, balancing the system between a doubly occupied localized vacancy state and a singly occupied vacancy with the other electron doping the conduction band. The latter case can also be viewed as a site-selective Mott insulator, in which the singly occupied in-gap state corresponds to the lower Hubbard band on the vacancy site.

\section{COMPUTATIONAL METHOD}

The key point in our analysis is the explicit inclusion of an electronic orbital corresponding to the oxygen vacancy point defect into the low-energy Hamiltonian constructed from the DFT results, together with the usual Ti- $t_{2 g}$ bands. This allows us to explore in detail the effect of the electronic correlations on the vacancy site, and it is the main novelty of the methodology presented here.

To obtain accurate geometries and initial band structures, we perform standard DFT calculations using the projectoraugmented-wave (PAW) method, as implemented in the Vienna Ab-initio Simulation Package (VASP) [47,48], version 5.4.1, together with the LDA exchange-correlation functional [49]. The valence configurations of the PAW potentials used are $\operatorname{Sr}\left(3 s^{2} 3 p^{6} 4 s^{2}\right)$, $\operatorname{Ti}\left(3 s^{2} 3 p^{6} 4 s^{1} 3 d^{3}\right)$, and $\mathrm{O}\left(2 s^{2} 2 p^{4}\right)$. To allow the system to accommodate the defect with a reasonable $\mathrm{O}_{\mathrm{V}}$ concentration, we use a 20-atom unit cell, 19-atom for the $\mathrm{O}_{\mathrm{V}}$-defective system $\mathrm{SrTiO}_{2.75}$, corresponding to an oxygen vacancy concentration of $8.3 \%$ [50]. Our calculations for $\mathrm{La}_{x} \mathrm{Sr}_{1-x} \mathrm{TiO}_{3}$ (Sec. III F) are performed using larger 40-atom supercells. For calculations of geometries and band structures (Secs. III A and III B), well-converged results are obtained by sampling the Brillouin zone with an $8 \times 8 \times 8 \Gamma$-centered $k$-mesh and using a plane-wave energy cutoff of $800 \mathrm{eV}$. For the more demanding cRPA calculations (Sec. IIID), the $k$-space sampling is done with a $4 \times 4 \times 4$ mesh, and an energy cutoff of $500 \mathrm{eV}$ is used. In Sec. III E ( Ti-O $\mathrm{O}_{\mathrm{V}}$-Ti distance dependence), these parameters are $8 \times 8 \times 8$ and $750 \mathrm{eV}$, and in Sec. III F (comparison with La doping), these are $4 \times 4 \times 4$ and $700 \mathrm{eV}$. Where lattice relaxation is employed, structural degrees of freedom are relaxed until forces fall below a $10^{-4}$ $\mathrm{eV} / \AA$ threshold, with the symmetry constrained to disable the rotations of the oxygen octahedra that occur in $\mathrm{SrTiO}_{3}$ below $\sim 100 \mathrm{~K}$. All calculations are performed with spin polarization excluded.

The low-energy correlated subspace for the DMFT calculations is then constructed using a basis of maximally localized Wannier functions (MLWF) [41,51], employing the WANNIER90 code [42]. We use the TRIQS/DFTTOOLS package [52-54] to implement the DMFT calculations, averaging over both spin channels to enforce a paramagnetic solution. An effective impurity problem is solved for each inequivalent Ti site plus the vacancy site using the TRIQS/CTHYB solver [55], while the different impurity problems are coupled through the DMFT self-consistency. The $\mathrm{O}_{\mathrm{V}}$ site is treated at the same level as the Ti sites, as introduced in Ref. [40] (more details about this implementation are given in Sec. IIIC). The DFT+DMFT calculations are performed without full charge self-consistency. The local interaction is modeled using the Hubbard-Kanamori parametrization with spin-flip and pair-hopping terms included [56]. Within the HubbardKanamori parametrization, the strength of the electronelectron interaction is described by the intraorbital Hubbard parameter $U$ and the Hund coupling parameter $J$. Note that these parameters are different from the average interaction parameters $\mathcal{U}$ and $\mathcal{J}$ typically used in DFT $+\mathcal{U}$ calculations. The double-counting correction is computed within the fully localized limit according to Held [57], and all calculations are performed at room temperature, $\beta=\left(k_{B} T\right)^{-1}=40 \mathrm{eV}^{-1}$. We use a fixed value of $J=0.64 \mathrm{eV}$ on the Ti sites, whereas the values for the Hubbard $U$, both on the vacancy and the Ti sites, are varied to analyze the effect on the electronic properties. Full frequency spectral functions, $A(\omega)$, are obtained from the local Green's functions in imaginary time, $G(\tau)$, using the Maximum Entropy algorithm [58]. The spectral weight 


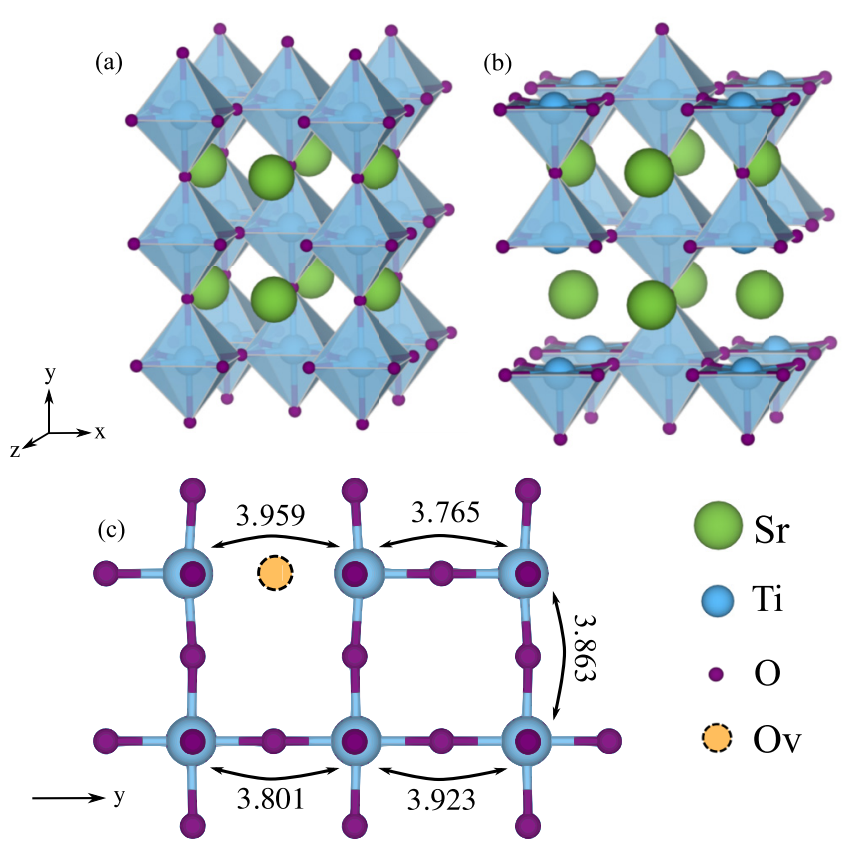

FIG. 1. Supercells of the calculated structures for (a) $\mathrm{SrTiO}_{3}$ (20-atom unit cell, $P m \overline{3} m$ symmetry) and (b) $\mathrm{SrTiO}_{3-\delta}$ (19-atom unit cell, $P 4 / \mathrm{mmm}$ symmetry). (c) Geometry of the relaxed structure containing an oxygen vacancy (indicated with the orange circle) with the Ti-Ti distances given in $\AA$. For comparison, the calculated Ti-Ti distance for stoichiometric $\mathrm{SrTiO}_{3}$ is $3.862 \AA$. Note that the orientation of part (c) is rotated relative to that of parts (a) and (b).

around the Fermi energy, $\bar{A}(0)$, is calculated from the impurity Green's function as $\bar{A}(0)=-\beta / \pi G(\beta / 2)$. The quasiparticle weight, $Z$, is calculated for each site as $Z=\left[1-\Sigma\left(i \omega_{0}\right)\right]^{-1}$, where $\Sigma\left(i \omega_{0}\right)$ is its self-energy at $\omega_{0}$, the smallest calculated Matsubara frequency.

We calculate the screened Coulomb interaction within the low-energy correlated subspace using the constrained random phase approximation (cRPA) [43-46]. In the cRPA method, a partial polarization function, $P_{r}$, is calculated by excluding all possible electronic transitions taking place within the correlated subspace. The bare Coulomb interaction, $v$, is then renormalized through screening by the higher-energy degrees of freedom through this $P_{r}$, yielding the frequencydependent partially screened Coulomb interaction $W_{r}(\omega)=$ $\left[1-v P_{r}(\omega)\right]^{-1} v$. The local interaction parameters of the Kanamori Hamiltonian, $U$ and $J$, are then obtained from the static limit by calculating matrix elements of $W_{r}(\omega=0)$, with the MLWFs used as local basis orbitals within the DMFT calculation, and taking appropriate averages over orbitals.

\section{RESULTS AND DISCUSSION}

\section{A. Geometry optimization}

Our calculated optimized LDA lattice constant for $\mathrm{SrTiO}_{3}$ constrained to cubic $P m \overline{3} m$ symmetry is $3.862 \AA$. [For a picture of the 20 -atom $(\sqrt{2}, 2, \sqrt{2})$ unit, see Fig. 1(a).] This value agrees well with literature LDA calculations [34], and it is not too far from the experimental value of $3.900 \AA$ [59]. In subsequent relaxations after removing one oxygen [Fig. 1(b)], we keep the lattice constants fixed to our calculated values for stoichiometric $\mathrm{SrTiO}_{3}$ [60]. The resulting structure and Ti-Ti distances are shown in Fig. 1(c). The removal of one of the oxygen atoms lowers the symmetry of the formerly cubic crystal to tetragonal $P 4 / \mathrm{mmm}$, in which the unique axis corresponds to the Ti- $\mathrm{O}_{\mathrm{V}}-\mathrm{Ti}$ chain $[y$ axis in Fig. 1(b)], and divides the Ti sites into two inequivalent types: one next to the vacancy, with five Ti-O bonds, and one farther from the vacancy, with a complete oxygen coordination octahedron. (Note that we do not include the low-temperature antiferrodistortive rotations of the oxygen octahedra; our preliminary tests suggest that their effect on the vacancy state is rather weak.) The Ti-Ti distances in the plane perpendicular to the tetragonal axis are slightly modified, with the vacancy pushing the two closest $\mathrm{Ti}$ apart, increasing their distance by $2.5 \%$ with respect to the distance in stoichiometric $\mathrm{SrTiO}_{3}$. The next Ti-O-Ti distance on the same axis contracts by the same amount, due to the volume constraint. On the other inequivalent Ti-O-Ti chain along the $y$ axis there is also a $1.6 \%$ contraction (expansion) of the Ti-O-Ti distance at the same (different) $y$ position as the closest $\mathrm{Ti}-\mathrm{O}_{\mathrm{V}}-\mathrm{Ti}$.

\section{B. DFT band structures}

The calculated band structures for stoichiometric $\mathrm{SrTiO}_{3}$ and defective $\mathrm{SrTiO}_{3-\delta}$ are shown and compared in Fig. 2. The low-energy region around the gap is composed of a valence band of mostly $\mathrm{O}-p$ character and a conduction band whose bottom part has mainly Ti- $t_{2 g}$ contributions, with some weight coming from the $\mathrm{O}-p$ orbitals, and minimal overlap with the Ti- $e_{g}$ and $\mathrm{Sr}-s$ bands at $\sim 3 \mathrm{eV}$ above the gap. Valence and conduction bands are separated by a gap of $1.8 \mathrm{eV}$, strongly underestimating the experimental value of $3.25 \mathrm{eV}$ [61]. The bands between approximately 0 and $3 \mathrm{eV}$ can be expressed in terms of MLWFs centered on the Ti atoms and showing a strong $t_{2 g}$ orbital character with additional admixtures of O- $p$ on the surrounding ligands; see Fig. 2(d).

The removal of one oxygen atom changes mainly the lower part of the conduction-band region of the band structure, with the appearance of an additional band that crosses the Fermi energy, $E_{F}$, making the system metallic, and accompanied by a lifting of degeneracies at the special points of the Brillouin zone. This band could accommodate, in principle, the two electrons released by the vacancy. However, its overlap with the bottom of the conduction-band edge (Fig. 2) results in a partial transfer of charge into the Ti- $t_{2 g}$ bands. Therefore, a complete description of the low-energy behavior of the system must include both the Ti- $t_{2 g}$ bands and this $\mathrm{O}_{\mathrm{V}}$-induced band, and we proceed by incorporating it into the minimal basis of Ti- $t_{2 g}$-like MLWFs in the DMFT treatment. We thus construct $12+1$ MLWFs, using initial $t_{2 g}$ projections on the Ti sites plus one $s$-like projection centered around the vacancy site.

The relationship of this new band to the $\mathrm{O}_{\mathrm{V}}$ is clearly seen from the MLWF centered on the position of the missing oxygen: its real-space representation shows an approximately spherical orbital centered around the vacancy with tails reaching to the neighboring ions [Fig. 2(e)]. Moreover, if one extracts a single MLWF centered on the vacancy site, the resulting Wannier band closely follows the new $\mathrm{O}_{\mathrm{V}}$-induced Bloch band [orange thick line in Fig. 2(b)]. As one can see 

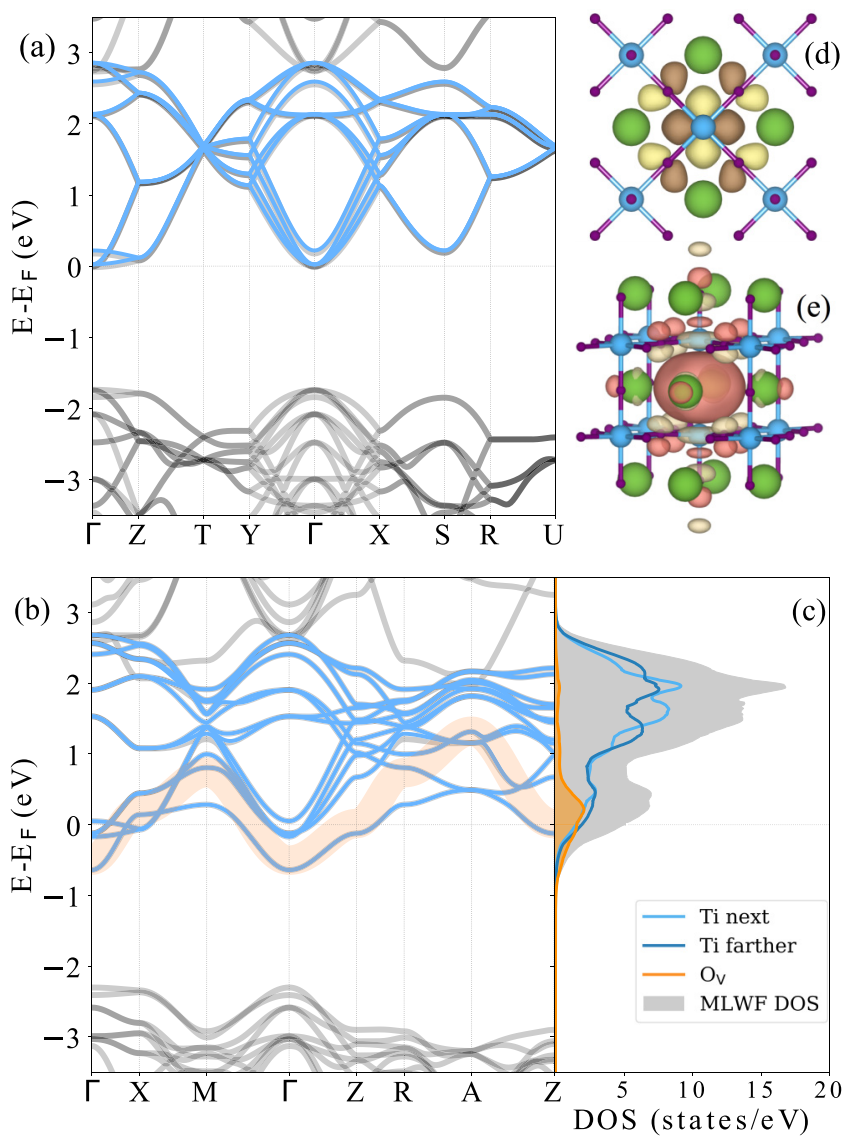

FIG. 2. Calculated DFT band structures for (a) stoichiometric $\mathrm{SrTiO}_{3}$ and (b) $\mathrm{SrTiO}_{2.75}$. DFT and MLWF bands are shown as gray and blue solid lines, respectively; the vacancy band in (b) is highlighted in orange (see the main text). (c) MLWF-projected density of states (DOS) for the $t_{2 g}$-like and $\mathrm{O}_{\mathrm{V}}$ MLWFs. Part (d) shows the real-space representation of a $d_{x z}$-type MLWF in the stoichiometric $\mathrm{SrTiO}_{3}$ system, while (e) corresponds to the $\mathrm{O}_{\mathrm{V}}$ MLWF.

from the MLWF-projected density of states [Fig. 2(c)], the $\mathrm{O}_{\mathrm{V}}$-centered MLWF accounts for most of the weight of this additional band. The occupations of the Wannier states, as calculated in DFT, are $0.21,0.28$, and 1.02 for the two inequivalent $\mathrm{Ti}$ sites, next to and farther from the $\mathrm{O}_{\mathrm{V}}$, and the $\mathrm{O}_{\mathrm{V}}$ site itself, respectively, in units of the electron charge.

\section{DMFT results}

Next, we perform DMFT calculations to investigate how an explicit local Hubbard-like interaction between the electrons affects the spectral properties and the charge distribution between the vacancy state and the Ti- $t_{2 g}$ bands. The input for the DMFT calculations is constructed from the tight-bindinglike Hamiltonian expressed in the basis of the MLWFs, plus the Coulomb matrix that models the electron-electron interaction. As stated in Sec. II, we simplify the latter for the $t_{2 g}$ states by using the Kanamori form, for which only two site-dependent parameters have to be specified: $U$, the on-site intraorbital Hubbard repulsion, and $J$, the Hund's coupling. For the $\mathrm{O}_{\mathrm{V}}$ site with only one orbital, there is only one parameter, $U$, describing the corresponding intraorbital Coulomb repulsion. This procedure allows us to independently vary the strength of the local interaction on the different types of sites, including the vacancy, and hence to determine the evolution of the system when $U\left(\mathrm{O}_{\mathrm{V}}\right)$ changes independently of $U(\mathrm{Ti})$. We note that for other early transition-metal perovskites, such as, e.g., $\mathrm{LaTiO}_{3}$, values of $U$ (Ti) between 4 and $5 \mathrm{eV}$ have often led to good agreement with experimental observations when using a minimal $t_{2 g}$ orbital subspace $[62,63]$. We therefore choose the range from 2 to $8 \mathrm{eV}$. On the other hand, given the lack of chemical intuition for choosing $U\left(\mathrm{O}_{\mathrm{V}}\right)$, we have considered $U\left(\mathrm{O}_{\mathrm{V}}\right)=0$ and $U\left(\mathrm{O}_{\mathrm{V}}\right)=U(\mathrm{Ti})$ as reasonable limits.

Figure 3 shows our calculated DMFT site occupations, along with the site-resolved quasiparticle weight $Z$, and the corresponding spectral functions for different choices of $U$ on the $\mathrm{Ti}$ and the $\mathrm{O}_{\mathrm{V}}$ sites. The top row [Fig. 3(a)] corresponds to $U\left(\mathrm{O}_{\mathrm{V}}\right)=0$, which is treating the electrons on the vacancy site as "uncorrelated." In this case, the effect of increasing $U$ (Ti) is to increase the $\mathrm{O}_{\mathrm{V}}$ occupation from $<1.5$ for small $U$ (Ti) to nearly 2 , which is almost completely filled, for large $U(\mathrm{Ti})$. For $U(\mathrm{Ti})>6 \mathrm{eV}$, a metal-insulator transition (MIT) is observed in which the Ti bands are totally depleted and the system becomes a band insulator. This MIT is related to a shift of the $\mathrm{O}_{\mathrm{V}}$ (Ti) spectral weight to lower (higher) energies until the corresponding overlap vanishes [see the spectral function for $U(\mathrm{Ti})=8 \mathrm{eV}$ ]. The quasiparticle weight $Z$ is close to 1 for all sites, independent of $U(\mathrm{Ti})$, indicating that the electrons remain uncorrelated, consistent with the transition from an uncorrelated metal to a band insulator [64]. Our DFT+DMFT result for higher $U(\mathrm{Ti})$ and $U\left(\mathrm{O}_{\mathrm{V}}\right)=0$ is thus equivalent to the results obtained in previous studies within DFT $+\mathcal{U}$ [32], as discussed in Sec. I.

The remaining rows in Figs. 3(b)-3(e) demonstrate the effect of introducing and then increasing a local Coulomb repulsion on the vacancy site, with $U\left(\mathrm{O}_{\mathrm{V}}\right) \leqslant U(\mathrm{Ti})$. By following the evolution of the $\mathrm{O}_{\mathrm{V}}$ site occupation as $U\left(\mathrm{O}_{\mathrm{V}}\right) / U(\mathrm{Ti})$ grows, we can see that the main effect of $U\left(\mathrm{O}_{\mathrm{V}}\right)$ is to first weaken the effect of $U$ (Ti) toward establishing a doubly occupied vacancy site [see the cases with $0<U\left(\mathrm{O}_{\mathrm{V}}\right) \leqslant U(\mathrm{Ti}) / 2$ in Figs. 3(b) and 3(c)], and then, for $U\left(\mathrm{O}_{\mathrm{V}}\right)>U(\mathrm{Ti}) / 2$, to drive the system instead toward a state with a half-filled $\mathrm{O}_{\mathrm{V}}$ and one electron doped into the Ti bands; see Figs. 3(d) and 3(e). In this limit, the Ti sites obtain an average filling of about 0.25 , with a slightly higher occupation of the Ti farther away from the vacancy.

Increasing $U\left(\mathrm{O}_{\mathrm{V}}\right)$ also strongly reduces the quasiparticle weight on the vacancy site, indicating strong local electronic correlations. Simultaneously, $U\left(\mathrm{O}_{\mathrm{V}}\right)$ triggers a reduction of $Z$ on the Ti sites, in particular on the site farther away from the vacancy. One can also observe a pronounced effect on the site-resolved spectral functions. In particular, increasing $U\left(\mathrm{O}_{\mathrm{V}}\right)$ results in a clear gap opening in the spectral function on the vacancy site once it reaches half-filling. This indicates a site-selective Mott transition, which is a localization of one electron on the vacancy site while the doped Ti bands remain metallic, albeit with a quasiparticle renormalization on the farther $\mathrm{Ti}$ site of $Z \approx 0.7$. Thus, this regime is characterized by a metallic conduction band doped with one electron per vacancy, and a split-off "in-gap" state containing one electron localized on the vacancy site. In the picture of the site-selective Mott insulator, this in-gap state corresponds to 
(a)

$\mathrm{U}\left(\mathrm{O}_{\mathrm{V}}\right)=0$

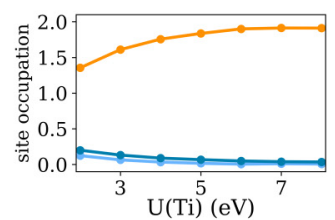

(b)

$\mathrm{U}\left(\mathrm{O}_{\mathrm{V}}\right)=\mathrm{U}(\mathrm{Ti}) / 4$

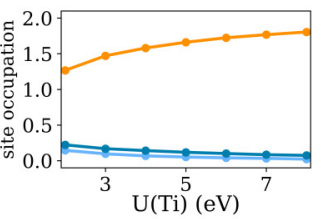

(c)

$\mathrm{U}\left(\mathrm{O}_{\mathrm{V}}\right)=\mathrm{U}(\mathrm{Ti}) / 2$

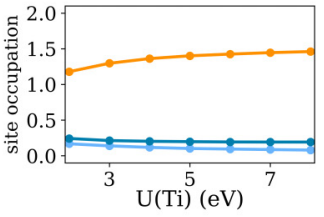

(d)

$\mathrm{U}\left(\mathrm{O}_{\mathrm{V}}\right)=3 \cdot \mathrm{U}(\mathrm{Ti}) / 4$

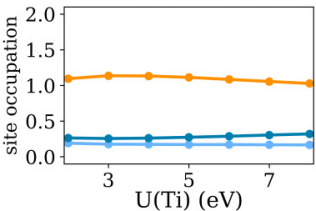

(e)

$\mathrm{U}\left(\mathrm{O}_{\mathrm{V}}\right)=\mathrm{U}(\mathrm{Ti})$

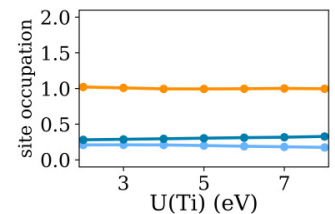

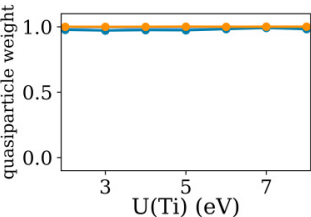
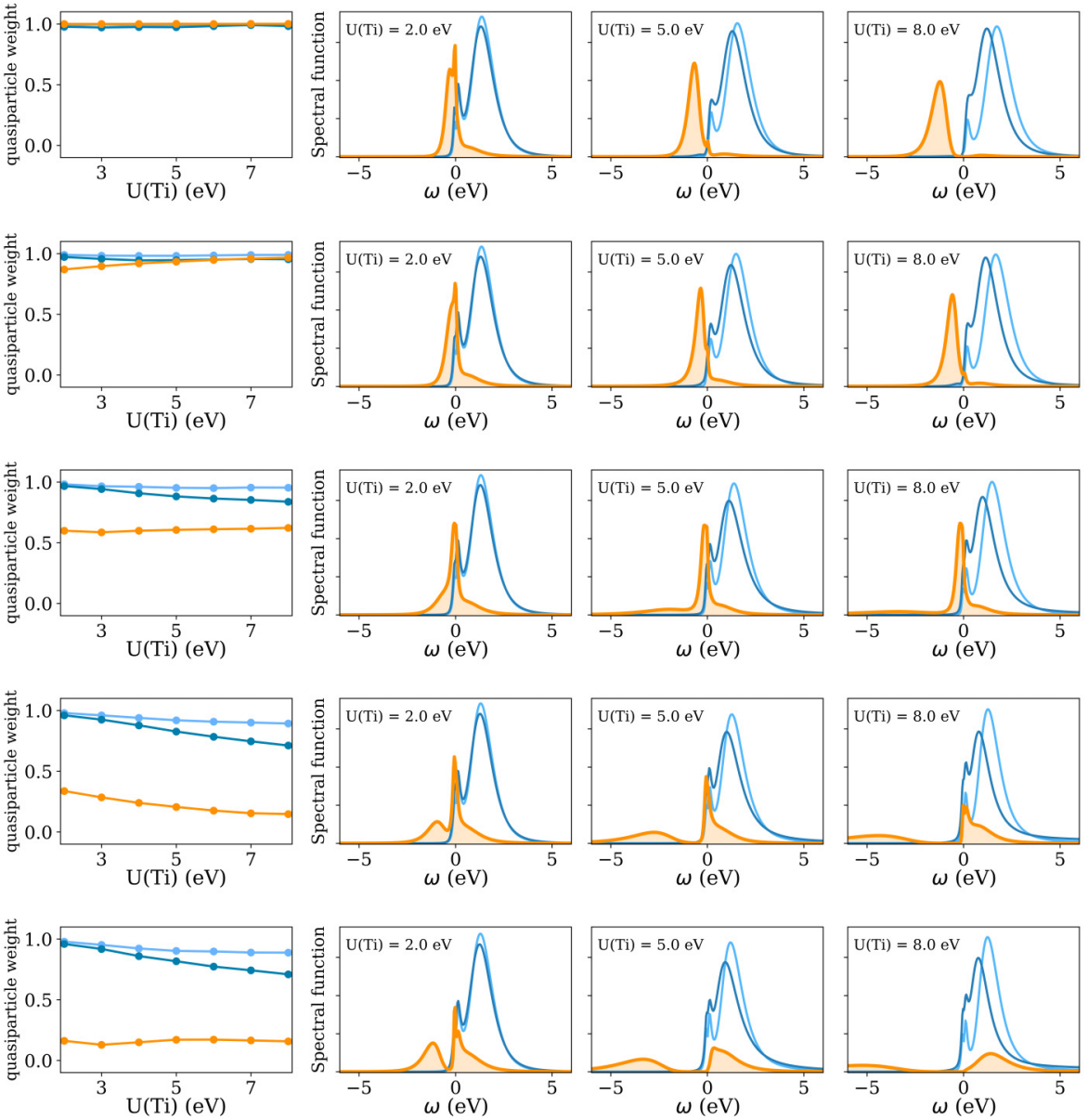

FIG. 3. DFT+DMFT results for different settings of $U\left(\mathrm{O}_{\mathrm{V}}\right) / \mathrm{U}(\mathrm{Ti})$, from 0 (a) to 1 (e). The left column in each row shows the site occupations for the three types of correlated sites: Ti-next, Ti-farther, and $\mathrm{O}_{\mathrm{V}}$ (lighter blue, darker blue and orange, respectively). The second to left column shows the corresponding quasiparticle weights $Z$. The following three plots in each row show the spectral functions for the three aforementioned sites for three different $U(\mathrm{Ti})$ values.

the lower Hubbard band of the vacancy site spectral function. Note that, in this regime of $U\left(\mathrm{O}_{\mathrm{V}}\right)=U(\mathrm{Ti})$, the filled region of the $\mathrm{O}_{\mathrm{V}}$ spectral function overlaps with the O- $p$ bands, which start $2 \mathrm{eV}$ below $E_{\mathrm{F}}$, but are excluded from the DMFT calculation.

Our results show that the specific treatment of electronic correlations on the $\mathrm{O}_{\mathrm{V}}$ site has a strong influence on whether the two electrons released by the missing oxygen are itinerant or localized. Several scenarios that have previously been discussed and reported for $\mathrm{SrTiO}_{3-\delta}$ can be realized with particular choices of $U(\mathrm{Ti})$ and $U\left(\mathrm{O}_{\mathrm{V}}\right)$, from the band-insulating limit with localization of the vacancy charge in the band gap [65] [high $U(\mathrm{Ti})$, zero $U\left(\mathrm{O}_{\mathrm{V}}\right)$ ] to the case of a paramagnetic impurity, in which one electron remains trapped in the gap while the other is delocalized into the Ti $d$-bands $\left[U(\mathrm{Ti})=U\left(\mathrm{O}_{\mathrm{V}}\right)\right][31]$. We note that the lack of charge selfconsistency in our calculations may diminish the quantitative nature of these results; however, the qualitative picture should remain valid.

\section{D. cRPA calculations}

The results discussed in the previous section show that the localization/delocalization of the two electrons associated with the missing oxygen depends critically on the values of the interaction parameters, $U(\mathrm{Ti})$ and $U\left(\mathrm{O}_{\mathrm{V}}\right)$. In some cases, values for the interaction parameters can be estimated from previous experience or from a comparison of certain calculated quantities with corresponding experimental measurements. In the present case, however, there is a lack of consensus among experimental studies (as described in Sec. I). Furthermore, due to the use of a nonstandard orbital basis, in particular on the vacancy site, it is not a priori clear what values for $U(\mathrm{Ti})$ and $U\left(\mathrm{O}_{\mathrm{V}}\right)$ will provide the most realistic description of $\mathrm{SrTiO}_{3-\delta}$. Therefore, we now estimate the strength of the screened Coulomb repulsion corresponding to our orbital basis using the constrained random phase approximation (cRPA) [43-46].

Within cRPA, the electronic degrees of freedom are divided into a "screening subspace" and a "target subspace," and excitations taking place exclusively within the target subspace are excluded from the screening (see Sec. II). Generally, the cRPA target subspace should be identical to the correlated subspace used for the DMFT calculation. However, there are also cases in which a certain number of "uncorrelated" bands are included in the DMFT subspace, without considering a local Hubbard-like interaction for these bands. In such cases, it might be appropriate to include these uncorrelated 


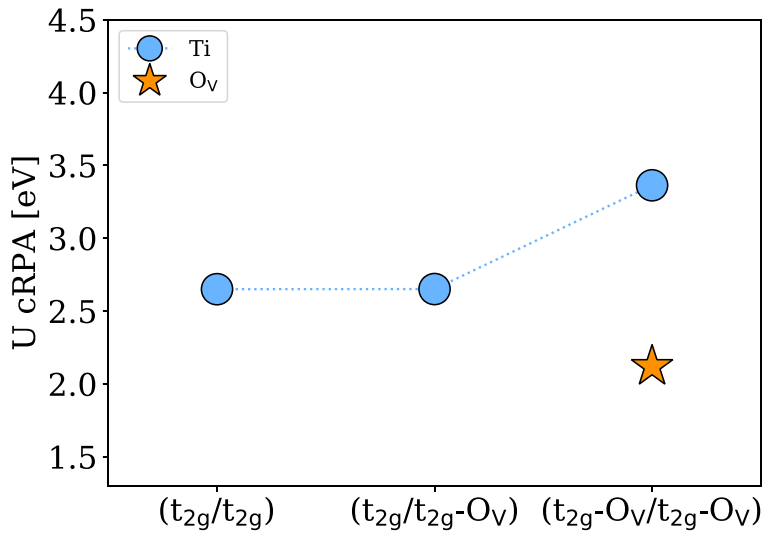

FIG. 4. Averaged values for the partially screened interaction parameters $U(\mathrm{Ti})$ and $U\left(\mathrm{O}_{\mathrm{V}}\right)$ obtained within cRPA. Blue dots represent the values for the Ti sites, while the orange star corresponds to the $\mathrm{O}_{\mathrm{V}}$ site. Displayed on the horizontal axis are the different schemes for choosing the target subspace (see the main text for more details).

bands within the screening subspace in the cRPA calculation $[44,45]$.

Thus, in order to gain additional insights, and to see how the calculated values depend on the specific subdivision of electronic degrees of freedom, we consider three different cases. In the first case, we construct only three $t_{2 g}$-like Wannier functions for each $\mathrm{Ti}$ site. These orbitals then also define the cRPA target subspace, achieved through a projection of the Bloch states onto these Wannier orbitals. All other bands, including that related to the vacancy, act as the screening subspace. Following the notation established in Ref. [44] and used in several other studies $[46,66,67]$, we denote this case as $\left(t_{2 g} / t_{2 g}\right)$. Here, the first symbol denotes the orbitals/bands defining the cRPA target subspace, while the second symbol indicates the whole set of Wannier functions that has been constructed. The screened interaction parameters are then evaluated for the Wannier functions spanning the cRPA target subspace.

For the second scenario, $\left(t_{2 g}-\mathrm{O}_{\mathrm{V}} / t_{2 g}-\mathrm{O}_{\mathrm{V}}\right)$, a full Wannier representation of the low-energy conduction bands in $\mathrm{SrTiO}_{3-\delta}$ containing three $t_{2 g}$-like Wannier functions per Ti plus an additional one located on the vacancy site is constructed, as described in the previous section. This whole set of Wannier functions is then used to define the cRPA target subspace. Thus, in this case the vacancy band is excluded from the screening and included in the target/correlated subspace.

The third, intermediate, case is denoted as $\left(t_{2 g} / t_{2 g}-\mathrm{O}_{\mathrm{V}}\right)$. Here, the same Wannier functions as in the previous case are constructed, but only the subset of $t_{2 g}$-like functions are used to define the cRPA target subspace. This essentially means that the effective interaction parameters calculated for the Ti- $t_{2 g}$ orbitals also include screening processes involving the vacancy band, similar to the first case, but using exactly the same Wannier representation as in the second scenario.

Figure 4 shows the values of the screened intraorbital interaction parameters $U(\mathrm{Ti})$ and $U\left(\mathrm{O}_{\mathrm{V}}\right)$, given by the corresponding averaged diagonal elements of the calculated $U$ tensor for all three cases. The difference in $U(\mathrm{Ti})$ between the

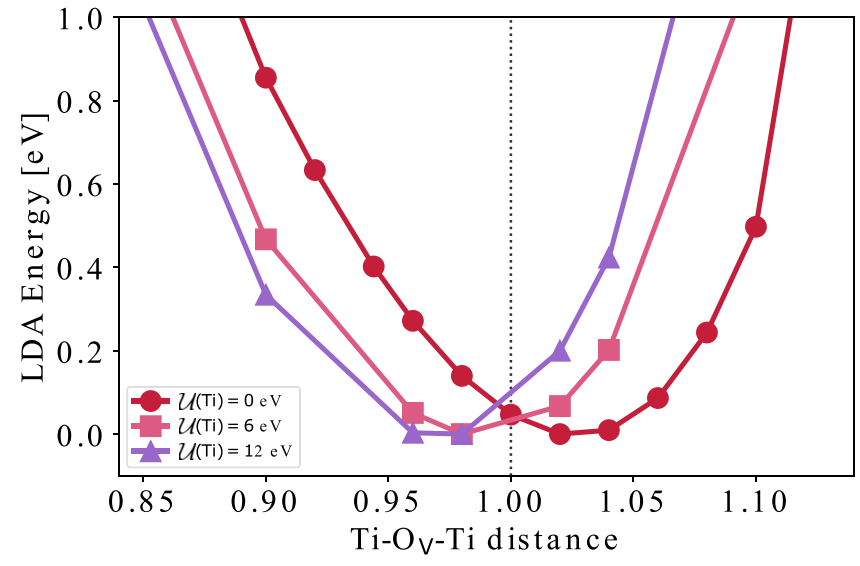

FIG. 5. Total energy as a function of the $\mathrm{Ti}-\mathrm{O}_{\mathrm{V}}-\mathrm{Ti}$ distance (in units of the Ti-O-Ti distance in stoichiometric $\mathrm{SrTiO}_{3}$ ) obtained within $\mathrm{LDA}+\mathcal{U}$ for three different choices of the Hubbard $\mathcal{U}$ parameter. The vertical line highlights the distance in cubic stoichiometric $\mathrm{SrTiO}_{3}$

$\left(t_{2 g} / t_{2 g}\right)$ and $\left(t_{2 g} / t_{2 g}-\mathrm{O}_{\mathrm{V}}\right)$ cases is negligible, in spite of the fact that the average quadratic spread of the Ti- $t_{2 g}$ Wannier orbitals is reduced from 3.3 to $2.0 \AA^{2}$ ( $4.9 \AA^{2}$ for the $\mathrm{O}_{\mathrm{V}}$ orbital). However, this merely increases the bare (unscreened) interaction parameter from 14.1 to $14.9 \mathrm{eV}$, indicating that the dominant factor in determining the final $U$ value is the screening. Thus, removing the vacancy band from the screening channel has a stronger effect, as can be seen by comparing cases $\left(t_{2 g} / t_{2 g}-\mathrm{O}_{\mathrm{V}}\right)$ and $\left(t_{2 g}-\mathrm{O}_{\mathrm{V}} / t_{2 g}-\mathrm{O}_{\mathrm{V}}\right)$, resulting in an increase of $U(\mathrm{Ti})$ from 2.65 to $3.47 \mathrm{eV}$, and demonstrating the sensitivity of $U$ on the specific screening channel. In addition, a sizable interaction parameter of $U\left(\mathrm{O}_{\mathrm{V}}\right)=2.12 \mathrm{eV}$ is obtained, which corresponds to approximately $60 \%$ of $U(\mathrm{Ti})$. This clearly shows that interaction effects cannot be neglected for the vacancy orbital, and that, in addition, the presence of the vacancy band strongly influences the effective interaction parameters on the Ti sites. These findings thus further support our approach of including an explicit treatment of correlation effects on the vacancy orbital, reinforcing the crucial role played by both $U(\mathrm{Ti})$ and $U\left(\mathrm{O}_{\mathrm{V}}\right)$ already presented in Sec. III C.

The values of the averaged interaction parameters obtained for the case $\left(t_{2 g}-\mathrm{O}_{\mathrm{V}} / t_{2 g}-\mathrm{O}_{\mathrm{V}}\right)$, namely $U(\mathrm{Ti}) \approx 3.5 \mathrm{eV}$ and $U\left(\mathrm{O}_{\mathrm{V}}\right) \approx 2.1 \mathrm{eV}$, would correspond to DFT+DMFT results with a vacancy occupation slightly larger than 1 and a corresponding quasiparticle weight below 0.5 , but still with a metallic spectral function on the vacancy site (Fig. 3). We note, however, that simply using the static $(\omega=0)$ value of the screened interaction in a DFT+DMFT calculation with frequency-independent local interaction might underestimate the corresponding interaction effects. Furthermore, recent work has shown that the random phase approximation can lead to an "overscreening" of the local interaction [68], and thus an underestimation of $U$, in particular for strongly correlated systems [69]. The cRPA results should thus rather be viewed as providing a rough ballpark, or a lower bound, rather than definite values to be used in realistic DFT+DMFT calculations [70]. 

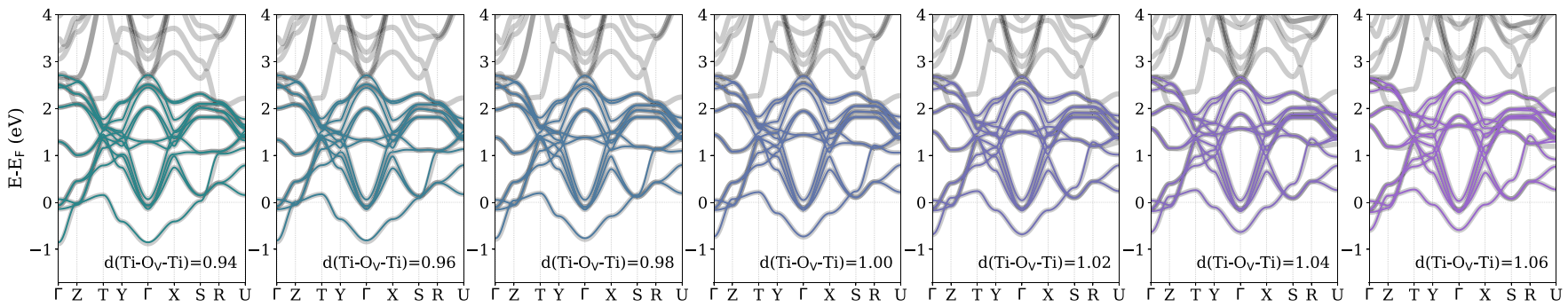

FIG. 6. Evolution of the $\mathrm{SrTiO}_{3-\delta}$ band structure with respect to the Ti- $\mathrm{O}_{\mathrm{V}}$-Ti distance, measured in units of the Ti-O-Ti distance in stoichiometric $\mathrm{SrTiO}_{3}$. The central plot corresponds to the Ti-O-Ti distance in stoichiometric $\mathrm{SrTiO}_{3}$. We see that bringing the two Ti ions closer together (left side) pushes the $\mathrm{O}_{\mathrm{V}}$ band down in energy, favoring charge localization, while pulling the Ti atoms apart (right side) enhances the entanglement of the $\mathrm{O}_{\mathrm{V}}$ band with the Ti- $t_{2 g}$ bands, and redistributes some of its charge onto the Ti ions.

\section{E. Influence of the $\mathrm{Ti}-\mathrm{O}_{\mathrm{V}}$-Ti distance}

As shown Fig. 1 and discussed in Sec. III A, structural relaxation using the standard LDA results in an outward relaxation of the two $\mathrm{Ti}$ atoms adjacent to the vacancy, and thus an elongation of the $\mathrm{Ti}-\mathrm{O}_{\mathrm{V}}-\mathrm{Ti}$ distance across the vacancy compared to the Ti-O-Ti distance in the ideal stoichiometric
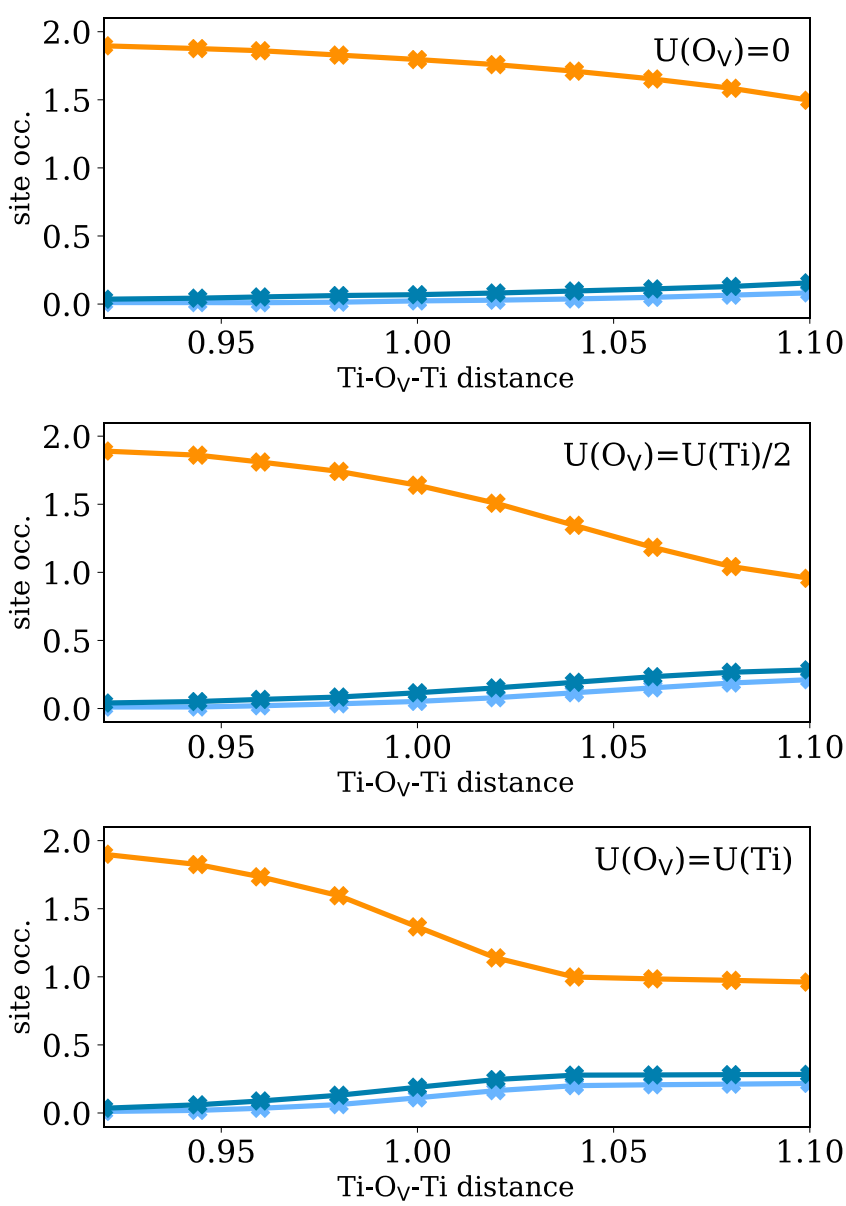

FIG. 7. $\mathrm{SrTiO}_{3-\delta}$ DMFT occupations for the three different sites as a function of the $\mathrm{Ti}^{-} \mathrm{O}_{\mathrm{V}}-\mathrm{Ti}$ distance (in units of the Ti$\mathrm{O}$-Ti distance in stoichiometric $\mathrm{SrTiO}_{3}$ ), for three different values of $U\left(\mathrm{O}_{\mathrm{V}}\right) / U(\mathrm{Ti})$. Lighter blue, darker blue, and orange represent Ti-next, Ti-farther, and $\mathrm{O}_{V}$ sites, respectively. Electron transfer from the $\mathrm{O}_{\mathrm{V}}$ to the $\mathrm{Ti}$ ions is favored by large $\mathrm{Ti}-\mathrm{O}_{\mathrm{V}}-\mathrm{Ti}$ distance and by large $U\left(\mathrm{O}_{\mathrm{V}}\right)$. case. While this is consistent with previous work using LDA or GGA $[71,72]$, other calculations using hybrid functionals or $\mathrm{LDA}+\mathcal{U}$ corrections have instead found a contraction of the $\mathrm{Ti}-\mathrm{O}_{\mathrm{V}}-\mathrm{Ti}$ distance, independent of other factors such as the size of the supercell [73,74]. As mentioned previously (see Sec. I), the latter calculations also find a tendency for charge localization, whereas LDA/GGA result in some charge spilling into the $\mathrm{Ti}$ bands. Therefore, we next manually tune the $\mathrm{Ti}^{-} \mathrm{O}_{\mathrm{V}}-\mathrm{Ti}$ distance and calculate the resulting changes in electronic properties.

Figure 5 shows the total energy calculated using LDA and $\mathrm{LDA}+\mathcal{U}$ for 19 -atom $\mathrm{SrTiO}_{3-\delta}$ as a function of the $\mathrm{Ti}-\mathrm{O}_{\mathrm{V}}-\mathrm{Ti}$ distance (in units of the Ti-O-Ti distance in stoichiometric $\mathrm{SrTiO}_{3}$ ). In these calculations, we only allow for a structural relaxation of those oxygen atoms that are situated next to the $\mathrm{Ti}$ in the same plane perpendicular to the $\mathrm{Ti}-\mathrm{O}_{\mathrm{V}}-\mathrm{Ti}$ direction. Calculations are performed for three different choices of the static Hubbard $\mathcal{U}$ parameter [75]. The case with $\mathcal{U}=0$ corresponds to a standard LDA calculation and leads to an increase of the Ti- $\mathrm{O}_{\mathrm{V}}$-Ti distance, consistent with the results presented in Sec. III A. Increasing $\mathcal{U}$ leads to a decrease of the relaxed $\mathrm{Ti}^{-} \mathrm{O}_{\mathrm{V}}-\mathrm{Ti}$ distance, in accordance with the aforementioned trend in the literature, ultimately shortening this distance with respect to that in pure $\mathrm{SrTiO}_{3}$.

To further explore the relationship between the localization of the defect state and the $\mathrm{Ti}-\mathrm{O}_{\mathrm{V}}$-Ti distance, we perform LDA calculations for a $\mathrm{SrTiO}_{3-\delta}$ structure in which we have systematically varied the $\mathrm{Ti}_{-} \mathrm{O}_{\mathrm{V}}-\mathrm{Ti}$ distance. For each (fixed) $\mathrm{Ti}_{\mathrm{V}} \mathrm{O}_{\mathrm{V}}-\mathrm{Ti}$ distance, the oxygen ions are allowed to relax within the tetragonal symmetry constraint, while all other atoms and the lattice constant are kept fixed. Figure 6 shows the evolution of the resulting DFT band structure as the $\mathrm{Ti}_{\mathrm{i}} \mathrm{O}-\mathrm{Ti}$ distance is varied over a range that includes also the various equilibrium values obtained for different values of $\mathcal{U}$ within $\mathrm{DFT}+\mathcal{U}$. We see that the $\mathrm{Ti}-\mathrm{O}_{\mathrm{V}}-\mathrm{Ti}$ distance affects primarily the position of the vacancy band, which is higher in energy, and therefore contains less electronic charge, at larger Ti- $\mathrm{O}_{\mathrm{V}}-\mathrm{Ti}$ distances. We conclude that short $\mathrm{Ti}-\mathrm{O}_{\mathrm{V}}-\mathrm{Ti}$ distances tend to localize the charge around the defect, while longer distances tend to partially delocalize the charge.

Following the same procedure as in Sec. III C, we then perform a series of DMFT calculations for the different structures corresponding to different $\mathrm{Ti}^{-} \mathrm{O}_{\mathrm{V}}-\mathrm{Ti}$ distances. We use $U(\mathrm{Ti})=4 \mathrm{eV}$ and three different values for $U\left(\mathrm{O}_{\mathrm{V}}\right)$ $[0, U(\mathrm{Ti}) / 2$, and $U(\mathrm{Ti})]$. Our calculated site occupations are displayed in Fig. 7. We see that for all values of $U\left(\mathrm{O}_{\mathrm{V}}\right)$, 

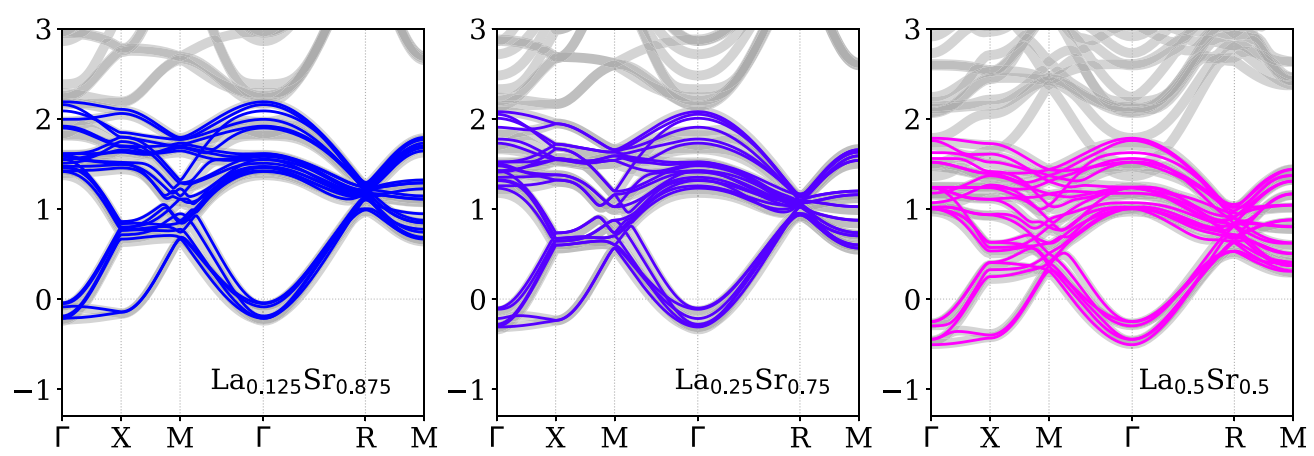

FIG. 8. LDA Band structure of $\mathrm{La}_{x} \mathrm{Sr}_{1-x} \mathrm{TiO}_{3}$ for different values of $x$. DFT and MLWF bands are shown in gray and in color (from blue to red), respectively. While the general shape and bandwidth of the Ti- $t_{2 g}$ remain basically constant for the whole series, its relative position with respect to $E_{F}$ moves gradually to lower energies for increasing $x$, marking the expected filling of the $t_{2 g}$ bands from $d^{0}$ for $\operatorname{SrTiO}_{3}(x=0)$ to $d^{1}$ in $\mathrm{LaTiO}_{3}(x=1)$.

the occupation of the vacancy is decreased with increasing $\mathrm{Ti}_{-} \mathrm{O}_{\mathrm{V}}-\mathrm{Ti}$ distance, while the $\mathrm{Ti}$ occupancies increase proportionally. The most pronounced change in occupation is observed for $U\left(\mathrm{O}_{\mathrm{V}}\right)=U$ (Ti) (Fig. 7, lower panel). In this case, the vacancy site occupation drops from close to 2 (for the shortest considered $\mathrm{Ti}^{-} \mathrm{O}_{\mathrm{V}}-\mathrm{Ti}$ distance of 0.92 ) down to 1 (for distances equal to or larger than 1.04). This case corresponds to the site-selective Mott-insulating state obtained previously in Sec. III C, in which the vacancy occupation is essentially locked to 1 due to the gap in the corresponding local spectral function. The strong dependence of the DMFT occupancies on $U\left(\mathrm{O}_{\mathrm{V}}\right)$ for larger $\mathrm{Ti}-\mathrm{O}_{\mathrm{V}}-\mathrm{Ti}$ distances is of course consistent with the results already presented in Fig. 3, where the structure was relaxed within $\mathrm{LDA}$, leading to a $\mathrm{Ti}^{-} \mathrm{O}_{\mathrm{V}}-\mathrm{Ti}$ distance of 1.024 in these units.

These results indicate that the site-selective Mottinsulating state, corresponding to a singly occupied vacancy site, is favorable for an elongated $\mathrm{Ti}-\mathrm{O}_{\mathrm{V}}-\mathrm{Ti}$ distance. We note that the lack of charge self-consistency does not allow us to extract reliable DFT+DMFT total energies as a function of the $\mathrm{Ti}-\mathrm{O}_{\mathrm{V}}-\mathrm{Ti}$ distance.

\section{F. Comparison with $\mathrm{La}_{\mathrm{Sr}}$ substitution}

As we mentioned in the Introduction, the question of how different doping sources affect the detailed electronic properties is an important one, with earlier studies suggesting that, while $\mathrm{O}_{\mathrm{V}}$ doping significantly alters the band structure as we have seen here, $\mathrm{La}_{\mathrm{Sr}}$ causes only a rigid shift of the bands $[20,76]$. Here, we test whether the DFT+DMFT treatment is consistent with these earlier findings by calculating the behavior when a small concentration of $\mathrm{Sr}$ ions is replaced by La.

Figure 8 shows the evolution of the DFT $\mathrm{La}_{x} \mathrm{Sr}_{1-x} \mathrm{TiO}_{3}$ band structure for three different values of $x$ corresponding to La concentrations of less than or equal to half. These results confirm that the main features of the $\mathrm{SrTiO}_{3}$ band structure are robust against doping. The only, yet rather important, difference between the three cases is the increasing partial filling of the Ti- $t_{2 g}$ bands with increasing La concentration.

The DFT+DMFT spectral functions of the Ti- $t_{2 g}$ bands for these three La concentrations, shown in Fig. 9(a), confirm the metallicity of the system. Their respective integrals up to $\omega=0$ yield the electron occupation of the corresponding orbitals, and in our simulations all have values consistent with a homogeneous distribution of the excess charge of one electron per La ion into the Ti bands. Increasing the strength of the interaction parameter from $U=4$ to $8 \mathrm{eV}$ does not have a noticeable effect on $A(\omega)$. However, the corresponding quasiparticle weights, $Z$ [Fig. 9(b)], are reduced from the "uncorrelated" value of $Z=1$, indicating some degree of electronic correlation in the corresponding bands. $Z$ decreases, and becomes more sensitive to the value of $U$, as the concentration of La increases, consistent with the proximity to the Mott-insulating state at $x=1$. Thus, our results confirm the validity of the rigid-band picture under $\mathrm{La}_{\mathrm{Sr}}$ substitution, while at the same time indicating moderate correlation effects, increasing with La concentration.

\section{SUMMARY AND CONCLUSIONS}

We have presented a DFT+DMFT description of the longdiscussed problem of oxygen vacancies in $\mathrm{SrTiO}_{3}$, focusing on a controlled and systematic treatment of electronic correlations on the vacancy site. This is achieved by using a minimal correlated subspace, which consists of the low-energy Ti- $t_{2 g}$ orbitals plus an additional Wannier function located on the vacancy site. Our study reveals a strong influence of $U\left(\mathrm{O}_{\mathrm{V}}\right)$ on the overall electronic structure of the system. Furthermore,
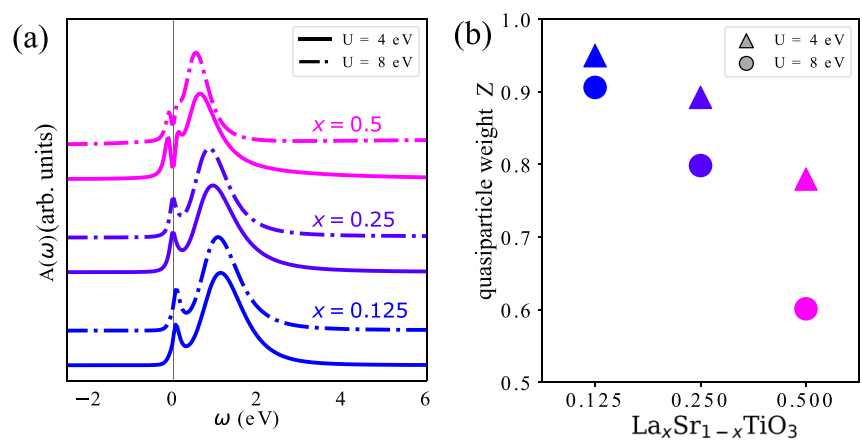

FIG. 9. (a) Ti- $t_{2 g}$ DMFT spectral functions and (b) corresponding quasiparticle weights for three different concentrations $x$ of $\mathrm{La}_{x} \mathrm{Sr}_{1-x} \mathrm{TiO}_{3}$ for a 40-atom unit cell and two different values of $U$. Spectral functions in (a) are shifted on the $y$-axis for clarity. 
our cRPA calculations support the importance of electronic correlations on the $\mathrm{O}_{\mathrm{v}}$ site, yielding a $U\left(\mathrm{O}_{\mathrm{v}}\right)$ close to $60 \%$ of $U$ (Ti).

In particular, we find a transition from a doubly occupied $\left[U\left(\mathrm{O}_{\mathrm{V}}\right)=0\right]$ to a singly occupied $\mathrm{O}_{\mathrm{V}}$ state $\left[U\left(\mathrm{O}_{\mathrm{V}}\right)=\right.$ $U(\mathrm{Ti})$ ], accompanied by a charge transfer of one electron from the defect state into the conduction band. The latter state is equivalent to the scenario proposed by Lin and Demkov based on a minimal model of the vacancy levels [38], and consistent with the spin-polarized GGA $+\mathcal{U}$ results of Hou and Terakura [31], but without the need for an artificial spinsymmetry breaking. It can in principle reconcile apparently contradictory experimental observations of low-temperature metallic conductivity in combination with localized in-gap states $[24,25,28,29]$. Our results are also consistent with a recent DFT+DMFT study of oxygen vacancies at the $\mathrm{SrTiO}_{3}$ surface [39], which also found metallicity in combination with a split-off spectral feature inside the gap.

Analysis of the effect of the distance between two Ti ions separated by an $\mathrm{O}_{\mathrm{V}}$ on the nature of the $\mathrm{O}_{\mathrm{V}}$ charge leads to two important conclusions. First, different treatments of electronic interactions at the DFT $(+\mathcal{U})$ level lead to different $\mathrm{Ti}-\mathrm{O}_{\mathrm{V}}-\mathrm{Ti}$ distances. This in turn affects the relative $\mathrm{O}_{\mathrm{V}}$ and Ti- $t_{2 g}$ energy levels, with smaller Ti- $\mathrm{O}_{\mathrm{V}}$-Ti distances favoring localization of the charge on the $\mathrm{O}_{\mathrm{V}}$ and larger distances promoting partial filling of the Ti orbitals with one of the electrons released by the missing oxygen. Second, this picture is largely maintained after a DFT+DMFT analysis, with the $\mathrm{O}_{\mathrm{V}}$ electron occupation also depending on $U\left(\mathrm{O}_{\mathrm{V}}\right)$. Future DFT+DMFT calculations considering full charge selfconsistency and structural relaxations could provide further insight as to which $\mathrm{Ti}^{-} \mathrm{O}_{\mathrm{V}}$-Ti distance and vacancy site occupation is indeed energetically preferred.
Lastly, we have compared $\mathrm{O}_{\mathrm{v}}$-doped $\mathrm{SrTiO}_{3}$ with the case of $\mathrm{La}_{\mathrm{Sr}}$ substitution, which represents another common way of electron doping the system. Our DFT+DMFT results, in line with previous DFT findings, show that $\mathrm{La}_{x} \mathrm{Sr}_{1-x} \mathrm{TiO}_{3}$ is much closer to the trivial doping case, with no significant change in the band structure other than an increasing uniform filling of the Ti- $t_{2 g}$ bands by the extra electrons donated by the La cations. This demonstrates that different routes to electrondoping in $\mathrm{SrTiO}_{3}$ are certainly not equivalent, and can lead to rather different electronic structures.

Finally, we mention that the method that we have demonstrated for controlling and analyzing the strength of electronic correlation on a vacancy defect state within the DFT+DMFT formalism is applicable to other defects beyond the oxygen vacancies studied here. Our finding that the electronic nature of the vacancy state depends strongly on the strength of the local interaction, $U\left(\mathrm{O}_{\mathrm{V}}\right)$, highlights the need for such a methodology. We hope that, in addition to contributing to the ongoing debate about the case of doped $\mathrm{SrTiO}_{3}$, our work will motivate similar studies on other materials in which correlated defects might play an important role.

\section{ACKNOWLEDGMENTS}

We thank Peitao Liu and Cesare Franchini for fruitful discussions and technical help with the cRPA calculations. This work was supported by the Swiss National Science Foundation through NCCR-MARVEL and by the Körber Foundation. Calculations have been performed on the cluster "Piz Daint," hosted by the Swiss National Supercomputing Centre and supported under project IDs s889 (User Lab) and mr26 (MARVEL), and the "Euler" cluster of ETH Zurich.
[1] D. Fuchs, C. W. Schneider, R. Schneider, and H. Rietschel, High dielectric constant and tunability of epitaxial $\mathrm{SrTiO}_{3}$ thin film capacitors, J. Appl. Phys. 85, 7362 (1999).

[2] J. H. Hao, Z. Luo, and J. Gao, Effects of substrate on the dielectric and tunable properties of epitaxial $\mathrm{SrTiO}_{3}$ thin films, J. Appl. Phys. 100, 114107 (2006).

[3] J. H. Barrett, Dielectric constant in perovskite type crystals, Phys. Rev. 86, 118 (1952).

[4] K. A. Müller and H. Burkard, $\mathrm{SrTiO}_{3}$ : An intrinsic quantum paraelectric below 4 K, Phys. Rev. B 19, 3593 (1979).

[5] J. F. Schooley, W. R. Hosler, and M. L. Cohen, Superconductivity in Semiconducting $\mathrm{SrTiO}_{3}$, Phys. Rev. Lett. 12, 474 (1964).

[6] C. Collignon, X. Lin, C. W. Rischau, B. Fauqué, and K. Behnia, Metallicity and superconductivity in doped strontium titanate, Annu. Rev. Condens. Matter Phys. 10, 25 (2019).

[7] X. Lin, Z. Zhu, B. Fauqué, and K. Behnia, Fermi Surface of the Most Dilute Superconductor, Phys. Rev. X 3, 021002 (2013).

[8] J. M. Edge, Y. Kedem, U. Aschauer, N. A. Spaldin, and A. V. Balatsky, Quantum Critical Origin of the Superconducting Dome in $\mathrm{SrTiO}_{3}$, Phys. Rev. Lett. 115, 247002 (2015).
[9] A. Ohtomo and H. Y. Hwang, A high-mobility electron gas at the $\mathrm{LaAlO}_{3} / \mathrm{SrTiO}_{3}$ heterointerface, Nature (London) 427, 423 (2004).

[10] A. Brinkman, M. Huijben, M. van Zalk, J. Huijben, U. Zeitler, J. C. Maan, W. G. van der Wiel, G. Rijnders, D. H. A. Blank, and $\mathrm{H}$. Hilgenkamp, Magnetic effects at the interface between non-magnetic oxides, Nat. Mater. 6, 493 (2007).

[11] J. N. Eckstein, Watch out for the lack of oxygen, Nat. Mater. 6, 473 (2007).

[12] D. D. Sarma, S. R. Barman, H. Kajueter, and G. Kotliar, Spectral functions in doped transition metal oxides, Europhys. Lett. 36, 307 (1996).

[13] W. Wunderlich, H. Ohta, and K. Koumoto, Enhanced effective mass in doped $\mathrm{SrTiO}_{3}$ and related perovskites, Phys. B 404, 2202 (2009).

[14] A. Kinaci, C. Sevik, and T. Çağın, Electronic transport properties of $\mathrm{SrTiO}_{3}$ and its alloys: $\mathrm{Sr}_{1-x} \mathrm{La}_{x} \mathrm{TiO}_{3}$ and $\mathrm{SrTi}_{1-x} M_{x} \mathrm{O}_{3}$ ( $M=\mathrm{Nb}, \mathrm{Ta})$, Phys. Rev. B 82, 155114 (2010).

[15] Y. Aiura, K. Ozawa, Y. Tezuka, M. Minohara, A. Samizo, K. Bando, H. Kumigashira, and K. Mase, In-gap state generated by La-on-Sr substitutional defects within the bulk of $\mathrm{SrTiO}_{3}$, Phys. Chem. Chem. Phys. 21, 14646 (2019). 
[16] T. Higuchi, T. Tsukamoto, K. Kobayashi, Y. Ishiwata, M. Fujisawa, T. Yokoya, S. Yamaguchi, and S. Shin, Electronic structure in the band gap of lightly doped $\mathrm{SrTiO}_{3}$ by highresolution X-ray absorption spectroscopy, Phys. Rev. B 61, 12860 (2000).

[17] S. Ohta, T. Nomura, H. Ohta, and K. Koumoto, Hightemperature carrier transport and thermoelectric properties of heavily La- or Nb-doped $\mathrm{SrTiO}_{3}$ single crystals, J. Appl. Phys. 97, 034106 (2005).

[18] J. D. Baniecki, M. Ishii, H. Aso, K. Kurihara, and D. Ricinschi, Density functional theory and experimental study of the electronic structure and transport properties of $\mathrm{La}, \mathrm{V}, \mathrm{Nb}$, and $\mathrm{Ta}$ doped $\mathrm{SrTiO}_{3}$, J. Appl. Phys. 113, 013701 (2013).

[19] Y. Tokura, Y. Taguchi, Y. Okada, Y. Fujishima, T. Arima, K. Kumagai, and Y. Iye, Filling Dependence of Electronic Properties on the Verge of Metal-Mott-Insulator Transition in $\mathrm{Sr}_{1-x} \mathrm{La}_{x} \mathrm{TiO}_{3}$, Phys. Rev. Lett. 70, 2126 (1993).

[20] N. Shanthi and D. D. Sarma, Electronic structure of electron doped $\mathrm{SrTiO}_{3}: \mathrm{SrTiO}_{3-\delta}$ and $\mathrm{Sr}_{1-x} \mathrm{La}_{x} \mathrm{TiO}_{3}$, Phys. Rev. B 57, 2153 (1998).

[21] A. Fujimori, I. Hase, M. Nakamura, H. Namatame, Y. Fujishima, Y. Tokura, M. Abbate, F. M. F. de Groot, M. T. Czyzyk, J. C. Fuggle, O. Strebel, F. Lopez, M. Domke, and G. Kaindl, Doping-induced changes in the electronic structure of $\mathrm{La}_{x} \mathrm{Sr}_{1-x} \mathrm{TiO}_{3}$ : Limitation of the one-electron rigid-band model and the Hubbard model, Phys. Rev. B 46, 9841 (1992).

[22] A. Fujimori, A. E. Bocquet, K. Morikawa, K. Kobayashi, T. Saitoh, Y. Tokura, I. Hase, and M. Onoda, Electronic structure and electron-phonon interaction in transition metal oxides with $d^{0}$ configuration and lightly doped compounds, J. Phys. Chem. Solids 57, 1379 (1996).

[23] O. N. Tufte and P. W. Chapman, Electron mobility in semiconducting strontium titanate, Phys. Rev. 155, 796 (1967).

[24] R. Moos and K. H. Hardtl, Defect chemistry of donor-doped and undoped strontium titanate ceramics between $1000^{\circ}$ and $1400^{\circ}$ C, J. Am. Ceram. Soc. 80, 2549 (1997).

[25] A. Ohtomo and H. Y. Hwang, Growth mode control of the free carrier density in $\mathrm{SrTiO}_{3-\delta}$ films, J. Appl. Phys. 102, 083704 (2007).

[26] R. Leonelli and J. L. Brebner, Time-resolved spectroscopy of the visible emission band in strontium titanate, Phys. Rev. B 33, 8649 (1986).

[27] T. Hasegawa, M. Shirai, and K. Tanaka, Localizing nature of photo-excited states in $\mathrm{SrTiO}_{3}$, J. Lumin. 87-89, 1217 (2000).

[28] D. Kan, T. Terashima, R. Kanda, A. Masuno, K. Tanaka, S. Chu, H. Kan, A. Ishizumi, Y. Kanemitsu, Y. Shimakawa, and M. Takano, Blue-light emission at room temperature from $\mathrm{Ar}^{+}$-irradiated $\mathrm{SrTiO}_{3}$, Nat. Mater. 4, 816 (2005).

[29] Y. Yamada, H. Yasuda, T. Tayagaki, and Y. Kanemitsu, Temperature Dependence of Photoluminescence Spectra of Nondoped and Electron-Doped $\mathrm{SrTiO}_{3}$ : Crossover from Auger Recombination to Single-Carrier Trapping, Phys. Rev. Lett. 102, 247401 (2009).

[30] M. L. Crespillo, J. T. Graham, F. Agulló-López, Y. Zhang, and W. J. Weber, Isolated oxygen vacancies in strontium titanate shine red: Optical identification of $\mathrm{Ti}^{3+}$ polarons, Appl. Mater. Today 12, 131 (2018).

[31] Z. Hou and K. Terakura, Defect states induced by oxygen vacancies in cubic $\mathrm{SrTiO}_{3}$ : First-principles calculations, J. Phys. Soc. Jpn. 79, 114704 (2010).
[32] D. D. Cuong, B. Lee, K. M. Choi, H.-S. Ahn, S. Han, and J. Lee, Oxygen Vacancy Clustering and Electron Localization in Oxygen-Deficient $\mathrm{SrTiO}_{3}: \mathrm{LDA}+U$ Study, Phys. Rev. Lett. 98, 115503 (2007).

[33] R. Evarestov, E. Blokhin, D. Gryaznov, E. A. Kotomin, R. Merkle, and J. Maier, Jahn-Teller effect in the phonon properties of defective SrTiO3 from first principles, Phys. Rev. B 85 , 174303 (2012).

[34] T. Tanaka, K. Matsunaga, Y. Ikuhara, and T. Yamamoto, First-principles study on structures and energetics of intrinsic vacancies in $\mathrm{SrTiO}_{3}$, Phys. Rev. B 68, 205213 (2003).

[35] X. Hao, Z. Wang, M. Schmid, U. Diebold, and C. Franchini, Coexistence of trapped and free excess electrons in $\mathrm{SrTiO}_{3}$, Phys. Rev. B 91, 085204 (2015).

[36] C. Ricca, I. Timrov, M. Cococcioni, N. Marzari, and U. Aschauer, Self-consistent DFT $+U+V$ study of oxygen vacancies in $\mathrm{SrTiO}_{3}$, Phys. Rev. Res. 2, 023313 (2020).

[37] The DFT $+\mathcal{U}$ treatment is applied to the Ti- $d$ shells, so only the small fraction of the $\mathrm{O}_{\mathrm{V}}$ weight that might get projected onto the $d$ orbitals, whatever the formalism chosen, will actually feel the localization effect.

[38] C. Lin and A. A. Demkov, Electron Correlation in Oxygen Vacancy in $\mathrm{SrTiO}_{3}$, Phys. Rev. Lett. 111, 217601 (2013).

[39] F. Lechermann, H. O. Jeschke, A. J. Kim, S. Backes, and R. Valentí, Electron dichotomy on the $\mathrm{SrTiO}_{3}$ defect surface augmented by many-body effects, Phys. Rev. B 93, 121103(R) (2016).

[40] J. Souto-Casares, N. A. Spaldin, and C. Ederer, DFT+DMFT study of oxygen vacancies in a Mott insulator, Phys. Rev. B 100, 085146 (2019).

[41] N. Marzari, A. A. Mostofi, J. R. Yates, I. Souza, and D. Vanderbilt, Maximally localized Wannier functions: Theory and applications, Rev. Mod. Phys. 84, 1419 (2012).

[42] A. A. Mostofi, J. R. Yates, G. Pizzi, Y.-S. Lee, I. Souza, D. Vanderbilt, and N. Marzari, An updated version of wannier90: A tool for obtaining maximally-localised Wannier functions, Comput. Phys. Commun. 185, 2309 (2014).

[43] F. Aryasetiawan, M. Imada, A. Georges, G. Kotliar, S. Biermann, and A. I. Lichtenstein, Frequency-dependent local interactions and low-energy effective models from electronic structure calculations, Phys. Rev. B 70, 195104 (2004).

[44] T. Miyake and F. Aryasetiawan, Screened Coulomb interaction in the maximally localized Wannier basis, Phys. Rev. B 77, 085122 (2008).

[45] T. Miyake, F. Aryasetiawan, and M. Imada, Ab initio procedure for constructing effective models of correlated materials with entangled band structure, Phys. Rev. B 80, 155134 (2009).

[46] L. Vaugier, H. Jiang, and S. Biermann, Hubbard $U$ and Hund exchange $J$ in transition metal oxides: Screening versus localization trends from constrained random phase approximation, Phys. Rev. B 86, 165105 (2012).

[47] G. Kresse and J. Furthmüller, Efficiency of ab-initio total energy calculations for metals and semiconductors using a plane-wave basis set, Comput. Mater. Sci. 6, 15 (1996).

[48] G. Kresse and D. Joubert, From ultrasoft pseudopotentials to the projector augmented-wave method, Phys. Rev. B 59, 1758 (1999).

[49] D. M. Ceperley and B. J. Alder, Ground State of the Electron Gas by a Stochastic Method, Phys. Rev. Lett. 45, 566 (1980). 
[50] This $\mathrm{O}_{\mathrm{V}}$ concentration would correspond to an electron density slightly larger than the typical highest values observed experimentally [25] (5\%, but it should be noted that they assumed two electrons per defect). Nevertheless, it gives a reasonable compromise between computational efficiency and a realistic model.

[51] F. Lechermann, A. Georges, A. Poteryaev, S. Biermann, M. Posternak, A. Yamasaki, and O. K. Andersen, Dynamical mean-field theory using Wannier functions: A flexible route to electronic structure calculations of strongly correlated materials, Phys. Rev. B 74, 125120 (2006).

[52] O. Parcollet, M. Ferrero, T. Ayral, H. Hafermann, I. Krivenko, L. Messio, and P. Seth, TRIQS: A toolbox for research on interacting quantum systems, Comput. Phys. Commun. 196, 398 (2015).

[53] M. Aichhorn, L. Pourovskii, P. Seth, V. Vildosola, M. Zingl, O. E. Peil, X. Deng, J. Mravlje, G. J. Kraberger, C. Martins, M. Ferrero, and O. Parcollet, TRIQS/DFTTools: A TRIQS application for $a b$ initio calculations of correlated materials, Comput. Phys. Commun. 204, 200 (2016).

[54] A set of scripts and tools can be found in https://github. com/materialstheory/soliDMFT.

[55] P. Seth, I. Krivenko, M. Ferrero, and O. Parcollet, TRIQS/CTHYB: A continuous-time quantum Monte Carlo hybridisation expansion solver for quantum impurity problems, Comput. Phys. Commun. 200, 274 (2016).

[56] C. Castellani, C. R. Natoli, and J. Ranninger, Magnetic structure of $\mathrm{V}_{2} \mathrm{O}_{3}$ in the insulating phase, Phys. Rev. B 18, 4945 (1978).

[57] K. Held, Electronic structure calculations using dynamical mean field theory, Adv. Phys. 56, 829 (2007).

[58] R. K. Bryan, Maximum entropy analysis of oversampled data problems, Eur. Biophys. J. 18, 165 (1990).

[59] L. Cao, E. Sozontov, and J. Zegenhagen, Cubic to tetragonal phase transition of $\mathrm{SrTiO}_{3}$ under epitaxial stress: An X-ray backscattering study, Phys. Status Solidi A 181, 387 (2000).

[60] Even though the presence of $\mathrm{O}_{\mathrm{V}}$ can cause an expansion with respect to the pure $\mathrm{SrTiO}_{3}$, the volume of $\mathrm{SrTiO}_{3-\delta}$ is kept fixed in order to prevent the interaction between periodic images of the unit cell.

[61] K. van Benthem, C. Elsässer, and R. H. French, Bulk electronic structure of $\mathrm{SrTiO}_{3}$ : Experiment and theory, J. Appl. Phys. 90, 6156 (2001).

[62] E. Pavarini, S. Biermann, A. Poteryaev, A. I. Lichtenstein, A. Georges, and O. K. Andersen, Mott Transition and Suppression of Orbital Fluctuations in Orthorhombic $3 d^{1}$ Perovskites, Phys. Rev. Lett. 92, 176403 (2004).

[63] K. Dymkowski and C. Ederer, Strain-induced insulator-to-metal transition in $\mathrm{LaTiO}_{3}$ within DFT+DMFT, Phys. Rev. B 89, 161109(R) (2014).
[64] Note that in the insulating state, the quantity we plot, $\left[1-\Sigma\left(i \omega_{0}\right)\right]^{-1}$, loses its meaning as approximate quasiparticle weight $Z$. Nevertheless, we include the corresponding data in our plots for consistency.

[65] V. E. Alexandrov, E. A. Kotomin, J. Maier, and R. A. Evarestov, First-principles study of bulk and surface oxygen vacancies in $\mathrm{SrTiO}_{3}$ crystal, Eur. Phys. J. B 72, 53 (2009).

[66] P. Seth, P. Hansmann, A. van Roekeghem, L. Vaugier, and S. Biermann, Towards a First-Principles Determination of Effective Coulomb Interactions in Correlated Electron Materials: Role of Intershell Interactions, Phys. Rev. Lett. 119, 056401 (2017).

[67] B. Amadon, T. Applencourt, and F. Bruneval, Screened Coulomb interaction calculations: cRPA implementation and applications to dynamical screening and self-consistency in uranium dioxide and cerium, Phys. Rev. B 89, 125110 (2014).

[68] C. Honerkamp, H. Shinaoka, F. F. Assaad, and P. Werner, Limitations of constrained random phase approximation downfolding, Phys. Rev. B 98, 235151 (2018).

[69] Q. Han, B. Chakrabarti, and K. Haule, Investigation into the inadequacy of cRPA in reproducing screening in strongly correlated systems, arXiv:1810.06116.

[70] Moreover, the $U$-regimes of the localization/delocalization boundary for the vacancy site might also change if charge self-consistency were included in the calculation.

[71] J. P. Buban, H. Iddir, and S. Öğüt, Structural and electronic properties of oxygen vacancies in cubic and antiferrodistortive phases of $\mathrm{SrTiO}_{3}$, Phys. Rev. B 69, 180102(R) (2004).

[72] W. Luo, W. Duan, S. G. Louie, and M. L. Cohen, Structural and electronic properties of $n$-doped and $p$-doped $\mathrm{SrTiO}_{3}$, Phys. Rev. B 70, 214109 (2004).

[73] D. Gryaznov, E. Blokhin, A. Sorokine, E. A. Kotomin, R. A. Evarestov, A. Bussmann-Holder, and J. Maier, A comparative $\mathrm{ab}$ initio thermodynamic study of oxygen vacancies in $\mathrm{ZnO}$ and $\mathrm{SrTiO}_{3}$ : Emphasis on phonon contribution, J. Phys. Chem. C 117, 13776 (2013).

[74] C. Mitra, C. Lin, J. Robertson, and A. A. Demkov, Electronic structure of oxygen vacancies in $\mathrm{SrTiO}_{3}$ and $\mathrm{LaAlO}_{3}$, Phys. Rev. B 86, 155105 (2012).

[75] S. L. Dudarev, G. A. Botton, S. Y. Savrasov, C. J. Humphreys, and A. P. Sutton, Electron-energy-loss spectra and the structural stability of nickel oxide: An LSDA+U study, Phys. Rev. B 57, 1505 (1998).

[76] Y. Ishida, R. Eguchi, M. Matsunami, K. Horiba, M. Taguchi, A. Chainani, Y. Senba, H. Ohashi, H. Ohta, and S. Shin, Coherent and Incoherent Excitations of Electron-Doped $\mathrm{SrTiO}_{3}$, Phys. Rev. Lett. 100, 056401 (2008). 